\title{
Abundancia relativa y patrones de actividad de Didelphis marsupialis en un área periurbana de Medellín, Colombia
}

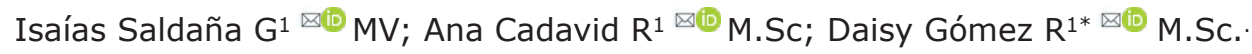

${ }^{1}$ Corporación Universitaria Remington. Facultad de Medicina Veterinaria. Grupo de investigación GINVER. Calle 51 Nro. 51-27. Medellín, Antioquia, Colombia. *Correspondencia: daisy.gomez@uniremington.edu.co

Recibido: Agosto 2018; Aceptado: Mayo 2019; Publicado: Septiembre 2019.

\section{RESUMEN}

Objetivo. Determinar la abundancia relativa y los patrones de actividad de la zarigüeya común (Didelphis marsupialis) en una zona periurbana del municipio de Medellín, departamento de Antioquia. Materiales y métodos. El estudio se desarrolló en la sede de prácticas y clínica veterinaria Remington ubicada en el corregimiento de Santa Elena. Para el registro de datos se empleó la metodología de fototrampeo durante 3 meses (junio-agosto de 2017), invirtiendo un esfuerzo total de 166 días/trampa. Resultados. Se obtuvieron un total de 275 registros independientes de la especie y se estimó una abundancia relativa de 52.88. El patrón de actividad muestra un primer pico de actividad al comienzo de la noche (19:00) y un segundo pico en la madrugada (3:00), con una posterior disminución hacia las horas de la madrugada. Conclusiones. Los resultados muestran una abundancia relativa alta con respecto a otras localidades en Colombia, además de evidenciar una población reproductivamente activa. Al mismo tiempo se convierten en una herramienta para el fortalecimiento de estrategias de conservación y sensibilización a los pobladores locales sobre la fauna de la región.

Palabras clave: Ecología, fauna silvestre, suburbano, zarigüeya (Fuente: AGROVOC, Tesauro ambiental para Colombia)

\section{ABSTRACT}

Objective. The relative abundance and activity patterns of the common opossum Didelphis marsupialis were determined in a peri-urban area of Medellín municipality, Antioquia department. Materials and methods. The study was developed in the Remington Veterinary clinic at Santa Elena Corregimiento. The data recording was carried out through camera trapping methodology for three months (June-August 2017) with a total effort of 166 days-camera. Results. With a total of 275 independent records, we calculate the relative abundance index (52.88 UNITS). The activity patterns show a first activity peak beginning the night (19:00) and a second one after midnight (3:00), reducing the activity in the sunrise hours. Conclusions. These results present a high relative abundance in comparison to another Colombian localities, besides a population active reproductively. At the same time, they become in a tool to support conservation strategies and sensibilization to local inhabitants about the wildlife of this region.

Keywords: Ecology, opossum, suburban areas, wildlife (Source: AGROVOC, Environmental thesaurus for Colombia). 


\section{INTRODUCCIÓN}

La urbanización alrededor de grandes concentraciones urbanas implica fuertes transformaciones del hábitat natural y la pérdida de la cobertura boscosa remanente, lo cual conlleva a un paisaje fragmentado con reducidas áreas naturales disponibles, generando efectos directos sobre la composición faunística de un área y contribuyendo a la pérdida de su biodiversidad local $(1,2)$. Estas transformaciones generan presiones adicionales sobre las poblaciones animales, obligando a los individuos a competir por el hábitat disponible y ocasionando efectos diferenciales sobre su supervivencia y abundancia (1). De esta manera, frente a dichos cambios algunas especies desaparecen o sus poblaciones decrecen, mientras otras son favorecidas por la aparición de nuevos recursos y los cambios en las interacciones ecológicas (3).

El estudio de la biodiversidad en zonas urbanas y periurbanas provee herramientas útiles para el diseño de estrategias de conservación dirigidas a promover la persistencia de las poblaciones de fauna y la conectividad entre fragmentos de cobertura boscosa (2). En este contexto, el estudio específico de especies tolerantes a la urbanización permite determinar y entender de manera directa los factores que facilitan la permanencia de la fauna en áreas intervenidas, y también obtener información básica para generar estrategias que promuevan la coexistencia de la fauna silvestre en ambientes urbanos.

La zarigüeya común (Didelphis marsupialis) es uno de los pocos mamíferos no voladores tolerantes a la transformación asociada a la urbanización. Esta especie no posee un riesgo inminente de extinción, debido a que se estima que su tamaño poblacional es grande y a su alta tolerancia a la transformación del hábitat, reflejada principalmente en su capacidad para usar recursos alternos en ambientes urbanos (i.e. alimento para animales domésticos y techos como nidos), (4,5). Sin embargo, en algunas zonas periurbanas la zarigüeya es aún percibida como un animal dañino e indeseable y en otras ha sido reportada como una de las especies con más altos índices de atropellamiento (6), lo que representa una amenaza adicional para la permanencia de sus poblaciones en este tipo ambientes.

La alta ocurrencia de las zarigüeyas en ambientes periurbanos y los servicios ambientales que puede proveer derivados del control de pequeños vertebrados y la dispersión de semillas (7), las convierten en un potencial modelo de estudio para entender los efectos de las transformaciones del hábitat y el tráfico vehicular sobre la fauna silvestre. El objetivo de este trabajo fue evaluar la abundancia relativa y los patrones de actividad de la zarigüeya común $D$. marsupialis en fragmentos de bosque secundario en una zona periurbana de alto tráfico vehicular en el Valle de Aburrá.

\section{MATERIALES Y MÉTODOS}

Área de estudio. El estudio fue desarrollado en la sede de prácticas veterinarias de la Corporación Universitaria Remington $\left(6.23907^{\circ} \mathrm{N}, 75.51438^{\circ}\right.$ O) ubicada en el corregimiento de Santa Elena, zona periurbana del municipio de Medellín (departamento de Antioquia). La sede cuenta con un área de $70.400 \mathrm{~m}^{2}$ y está compuesta por terrenos destinados al pastoreo y algunos fragmentos de bosque secundario altamente intervenidos, los cuales son delimitados por vías de alto flujo vehicular (Figura 1). El área se localiza sobre los 2.300 msnm y corresponde a la zona de vida de bosque muy húmedo montano bajo (bmhMB; Holdridge 1964).

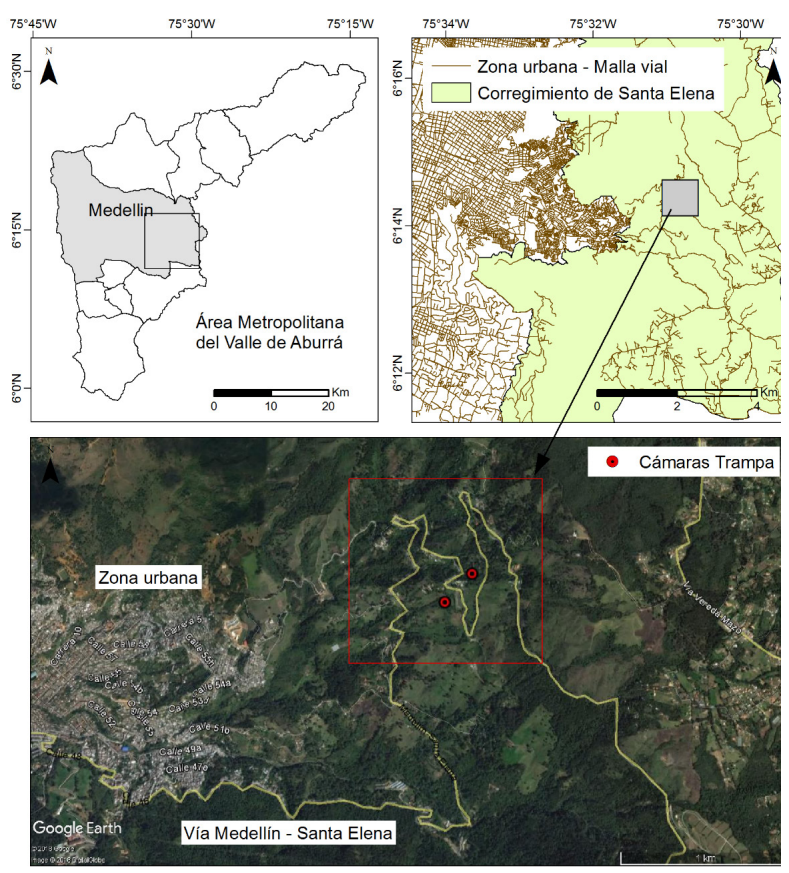

Figura 1. Localización geográfica del área de estudio en el corregimiento de Santa Elena, Medellín, Colombia.

Métodos. Para el registro de D. marsupialis se instalaron dos cámaras trampa Bushnell Trophy Cam HD en cada uno de dos fragmentos de bosque presentes en el área de estudio (Figura 1). Las cámaras fueron fijadas a árboles a una altura no mayor de $30 \mathrm{~cm}$ del suelo y ubicadas sobre senderos cercanos a cuerpos de agua en los que se identificó la presencia de rastros de fauna. A lo largo de todo el muestreo se empleó una 
configuración para la grabación de vídeos de $20 \mathrm{~s}$ a intervalos de $40 \mathrm{~s}$. Las cámaras permanecieron instaladas entre el 6 de junio y el 28 de agosto de 2017, acumulando un esfuerzo total de muestreo de 166 días/trampa.

Durante todo el período de muestreo las cámaras fueron cebadas con dos tipos de cebos. El primero correspondió a una conserva enlatada de pescado en salsa de tomate, la cual fue agujereada y fijada durante todo el tiempo de muestreo al árbol de instalación de la cámara. El segundo tipo estuvo compuesto de una mezcla de cereales, granos y mantequilla de maní que fue dejada en bolos de aproximadamente $200 \mathrm{~g}$ sobre el suelo al frente de la cámara. Esta mezcla era renovada cada semana durante el proceso de revisión y recolección de información con el fin de maximizar el número de registros $(8,9)$.

Análisis de datos. Para determinar el patrón de actividad y estimar los valores de abundancia de la especie se utilizaron únicamente registros independientes, empleando como criterio de selección un intervalo mayor a $30 \mathrm{~min}$ entre registros $(10,11)$. El patrón de actividad diario fue generado a partir de las horas de registro de cada vídeo mediante el método de curvas de densidad de Kernel (12). Este análisis fue realizado con el paquete Overlap en el programa R versión 3.4.3. La abundancia relativa fue estimada como el número de registros independientes sobre el número total de registros del muestreo por un factor de corrección 100 (trampas/noche) (13). Adicionalmente, para permitir las comparaciones con estudios previos sobre la especie, se estimó el índice de abundancia relativa como el número de registros independientes por cada 100 días de trampas $(11,14)$. Este índice también es denominado como frecuencia de detección y puede ser interpretado como una medida del éxito de muestreo (14).

\section{RESULTADOS}

A lo largo del tiempo de muestreo se obtuvieron un total de 520 registros, incluyendo mamíferos silvestres, fauna doméstica, algunas aves y 275 registros independientes de $D$. marsupialis. Los registros de la especie correspondieron a individuos de ambos sexos con diferencias notables de tamaño y condición reproductiva. Las diferencias en tamaño permitieron la distinción entre juveniles y adultos, y la actividad reproductiva de las hembras fue evidenciada a través de individuos con marsupios visibles, posiblemente albergando crías, e individuos con crías sobre la espalda (Figura 2).

A partir de los registros independientes se estimó una abundancia relativa de 52.88 para la especie en el área y un índice de abundancia relativa o frecuencia de detección de 165.66 fotos/100 trampas noche. El patrón de actividad fue exclusivamente nocturno, sin ninguna actividad durante el período de luz, y caracterizado por dos picos máximos en el período
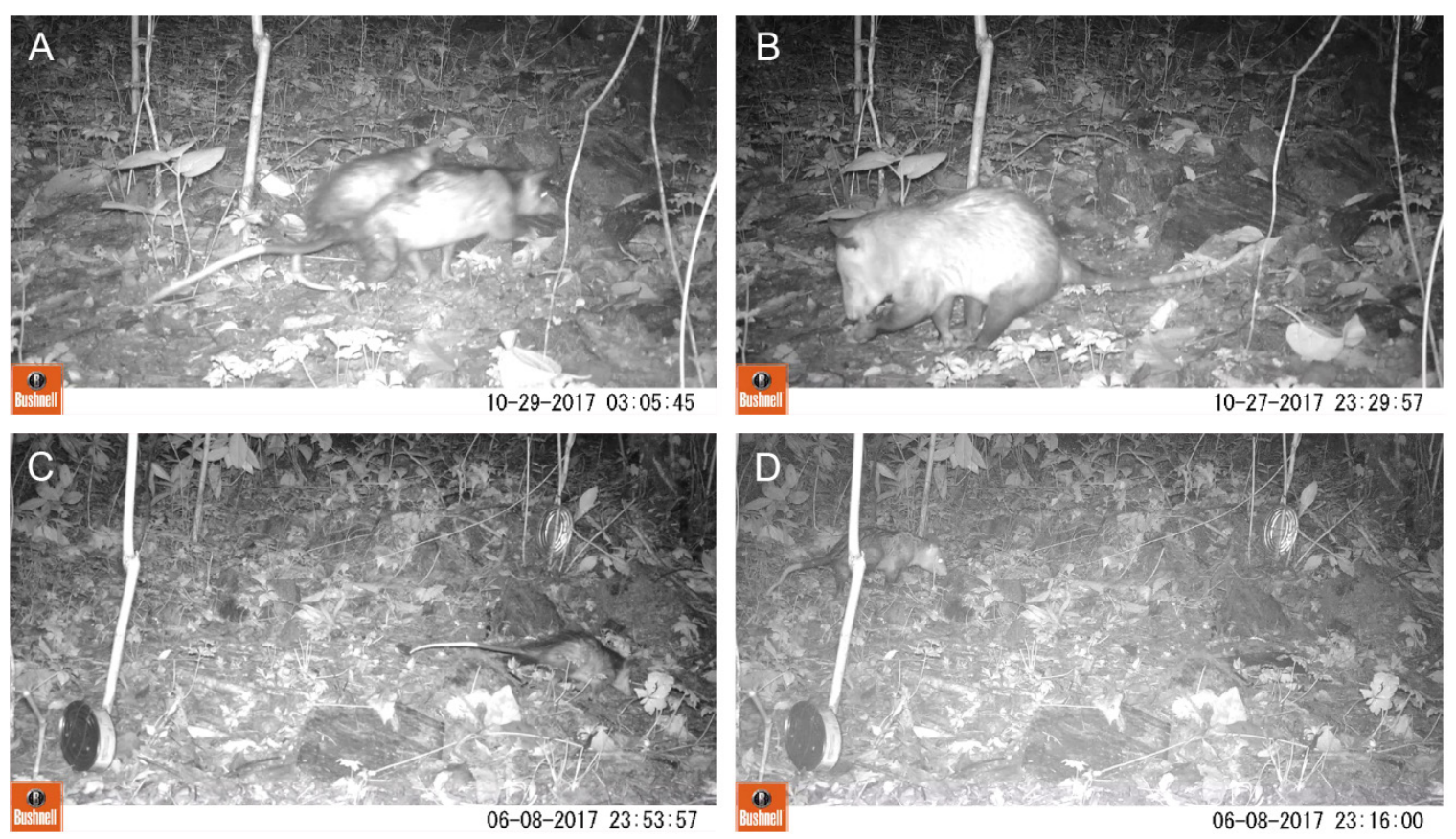

Figura 2. Registros de Didelphis marsupialis A. Hembra con cría en la espalda. B. Adulto alimentándose. C. Juvenil. D. Adulto. 
noche-madrugada (Figura 3). El primer pico es observado entre las 19:00 y 20:00 h, en el cual la densidad de actividad alcanza el valor máximo de 0.14 . Posteriormente la actividad decrece y fluctúa hacia la media noche, y un segundo pico máximo de actividad es observado entre las 2:00 y 3:00 h (densidad de actividad=0.11), después de lo cual la actividad disminuye dramáticamente hacia las 6:00 h (Figura 3).

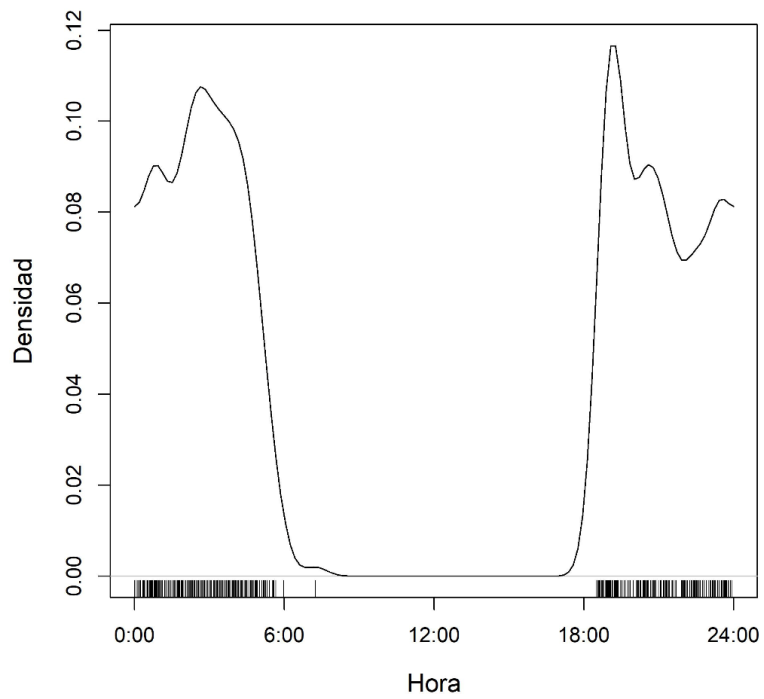

Figura 3. Patrones de actividad de la zarigüeya común $D$. marsupialis estimados mediante densidades de Kernel.

\section{DISCUSIóN}

Las estimaciones de abundancia relativa en este estudio fueron considerablemente mayores a las reportadas para otras zonas del país. La abundancia relativa de $D$. marsupialis ha sido estimada cercana a 13 en el piedemonte de los Farallones de Cali (esfuerzo de 2488 días/ trampa) (8), y para zonas del valle de Aburrá se han reportado índices de abundancia de 0.18 (corregimiento de Santa Elena) y 0.40 fotos/100 noche trampa (municipio de Caldas) con esfuerzos de muestreo de 360 días/trampa y 38 días/ trampa respectivamente (9). Aunque no es posible estimar el número de individuos en nuestra área de estudio, las estimaciones de abundancia y los registros obtenidos sugieren que la zona posiblemente mantiene una población estable y reproductivamente activa de $D$. marsupialis.

Aunque $D$. marsupialis es una especie comúnmente registrada en estudios de diversidad, su abundancia relativa ha sido poco estimada para el país. Empleando la metodología de trampas huella se ha reportado a la especie asociada principalmente a coberturas secundarias, presentando abundancias mayores en zonas de pastizales y cultivos (15) en ecosistema de bosque seco tropical. Mientras que, con trampas de captura viva, se estimó la abundancia relativa en zonas de bosque secundario inmerso entre cultivos en el norte de Colombia (16).

El patrón de actividad estrictamente nocturno encontrado para la especie en este trabajo coincide con lo reportado para poblaciones en otras zonas periurbanas del país (8), y para poblaciones en áreas naturales en otras zonas del Neotrópico (Costa Rica) (17). Asimismo, el patrón es similar a los perfiles de actividad documentados para las especies del género $D$. pernigra (18) y $D$. aurita (19). Aunque D. marsupialis es una especie ampliamente distribuida en el país (5), nuestros resultados se constituyen en el segundo esfuerzo por abordar específicamente los patrones de actividad de la especie en ambiente periurbanos, como una base para el entendimiento de los factores ecológicos que permiten su permanencia en remanentes de bosque urbanos.

Consideramos que los valores de abundancia obtenidos en este estudio deben tomarse con precaución, ya que las condiciones del paisaje y del muestreo pueden haber generado una sobrestimación. Aunque D. marsupialis puede hacer uso de diversas coberturas vegetales, la disponibilidad óptima del hábitat para la especie en la zona de estudio es reducida y está limitada a fragmentos de bosque que no superan los $15 \mathrm{~m}$ de ancho, lo cual podría obligar a los individuos presentes a usar con mayor intensidad la misma área. El monitoreo de un área pequeña, con hábitat reducido, además del uso de un atrayente en las trampas y un bajo número de unidades de muestreo, claramente generó un incremento en la probabilidad de detección de la especie y un aumento directo en las estimaciones de abundancia. Bajo estas condiciones, sugerimos que la abundancia relativa y la frecuencia de detección estimada, sean consideradas más como una aproximación a la probabilidad de uso que la especie hace del área y no como una aproximación a una variable de estado de la población.

Aunque el presente estudio sólo estima la abundancia relativa en un tiempo corto, permite establecer una línea base para la especie en áreas intervenidas del valle de Aburrá, abriendo la posibilidad a preguntas de investigación sobre la dinámica de las poblaciones y sus respuestas a la presión antrópica. Ésta información es de importancia en escenarios de conservación local, donde estimaciones sobre variables poblacionales permiten reconocer los impactos reales de problemáticas como el atropellamiento vehicular que afectan a la especie en Antioquia (6). 
Adicionalmente, es importante considerar que la aplicación de estas estimaciones debe ser considerada con cautela y entendida bajo el contexto del paisaje regional, con el fin de evitar conclusiones erróneas sobre la abundancia real de las especies. La razón es que en pequeños fragmentos puede estar ocurriendo una sobreestimación de variables poblacionales, asociado a un efecto del tamaño del hábitat disponible, como se ha reportado para otras especies de mamíferos (20).

\section{Conflicto de intereses.}

Los autores del presente estudio declaramos que no existe conflicto de intereses con la publicación de este manuscrito.

\section{Agradecimientos}

Agradecimos a la Corporación Universitaria Rémington por la financiación del estudio, al grupo de investigación GINVER y al semillero de fauna silvestre UFASI. A Camilo Sánchez Giraldo y dos revisores anónimos por sus valiosos aportes durante la escritura del manuscrito.

\section{REFERENCIAS}

1. McKinney, M. Effects of urbanization on species richness: A review of plants and animals. Urban Ecosyst. 2008; 11(2):161176. doi: 10.1007/s11252-007-0045-4. https://doi.org/10.1007/s11252-007-0045-4

2. Marin M, Alvarez F, Giraldo C, Pyrcz T, Uribe $S$, Vila R. Butterflies of an andean periurban cloud forest in the Aburra valley, Colombia. Rev Mex Biodivers. 2014; 85(1):200208. doi: $10.7550 /$ rmb.36605. https://doi. org/10.7550/rmb.36605

3. Bradley C, Altizer S. Urbanization and the ecology of wildlife diseases. Trends Ecol Evol. 2006; 22(2):95-102. doi: 10.1016/j. tree.2006.11.001. https://doi.org/10.1016/j. tree.2006.11.001

4. Astua D, Lew D, Costa L, Pérez-Hernandez R. Didelphis marsupialis [Internet]. The IUCN red list of threatened species. 2016 [citado 2 de septiembre de 2017]. doi: 10.2305/iucn. uk.2016-1.rlts.t40501a22176071.en https:// doi.org/10.2305/IUCN.UK.2016-1.RLTS. T40501A22176071.en

5. Solari S. Didelphis marsupialis. En: SánchezLondoño J, Marín-C D, Botero-Cañola S, Solari S. eds. Mamíferos silvestres del Valle de Aburra. Área metropolitana del Valle de Aburra, Corantioquia. Universidad de Antioquia: Colombia; 2014. ISBN 978-958.8513-79-9. https://acuareladelmundo.com/2015/06/20/ imama-mamiferos-silvestres-del-valle-deaburra/
6. Delgado-V C. Muerte de mamíferos por vehículos en la vía del Escobero, Envigado (Antioquia), Colombia. Actu Biol 2007; 29(87):229-233. https://aprendeenlinea. udea.edu.co/revistas/index.php/actbio/ article/view/329342/20785822

7. Franco-Quimbay J, Rojas-Robles R. Frugivoría y dispersión de semillas de la palma Oenocarpus bataua en dos regiones con diferente estado de conservación. Actu Biol 2004; 37(102):273-285. https:// aprendeenlinea.udea.edu.co/revistas/index. php/actbio/article/view/329005

8. Mosquera M, Corredor G, Cardona P, Armbrecht I. fototrampeo de aves caminadoras y mamíferos asociados en el pidedemonte de farallones de Cali. Bol Cient Mus Hist Nat. 2015; 18(2):144-156. https://www. thefreelibrary.com/Fototrampeo+de+aves+ caminadoras $+y+$ mamiferos+asociados+en +el...-a0451148391

9. Cabrera J, Galeano R, Mazabel R, Quintana D, Monsalve B. Evaluación del estado actual de zarigüeyas (Didelphis marsupialis) en tres zonas del valle de Aburrá. J Agric Anim Sci. 2017; 6(1):30-40. https://doi.org/10.22507/ jals.v6n1a3

10. González-Maya J, Schipper G, Benítez A. Activity patterns and community ecology of small carnivores in the Talamanca region, Costa Rica. Small Carniv Conserv. 2009; 41:9-14. http://nebula.wsimg.com/b32922 2e5d557b18e18cf81bc29bb639?AccessKeyI $\mathrm{d}=35 \mathrm{E} 369 \mathrm{A09ED} 705622 \mathrm{D} 78$ \&disposition $=0$ \&alloworigin $=1$ 
11. O'brien T, Kinnaird M, Wibisono H. Crouching tigers, hidden prey: sumatran tiger and prey populations in a tropical forest landscape. Anim Conserv. 2003; 6:131-139. doi: https:// doi.org/10.1017/S1367943003003172

12. Ridout M, Linkie M. Estimating overlap of daily activity patterns from camera trap data. J Agric Biol Environ Stat. 2009; 14(3):322-337. doi: https://doi.org/10.1198/jabes.2009.08038

13. Díaz-Pulido A, Payán E. Manual de fototrampeo: una herramienta de investigación para la conservación de la biodiversidad en Colombia. Instituto de Investigaciones de Recursos Biológicos Alexander von Humboldt y Panthera Colombia: Colombia; 2012. http://repository.humboldt.org.co/ bitstream/handle/20.500.11761/31415/240. pdf? sequence $=1$ \&isAllowed $=y$

14. Jiménez-Alvarado J, Moreno-Díaz $C$, Alfonso A, Giordano A, Vela-Vargas M, Gómez-Hoyos $D$, et al. Ciudades biodiversas: mamíferos medianos de la reserva forestal protectora bosque oriental de Bogotá, D.C. Colombia. Mammalogy Notes. 2017; 4(1):37-41. https://docs.wixstatic.com/ugd/f964e0 d0f6bd7c3cc6408081d7fc55b4d7012d.pdf

15. Orjuela C, Jimenez G. Estudio de la abundancia relativa para mamíferos en diferentes tipos de coberturas y carretera, finca hacienda cristales, área Cerritos - La Virginia, municipio de Pereira, departamento de Risaralda - Colombia. Univ Sci. 2004; 9:87-96. https://revistas.javeriana.edu.co/index.php/ scientarium/article/view/5028
16. Adler G, Arboledo J, Travi B. Population dynamics of Didelphis marsupialis in northern Colombia. Stud Neotrop Fauna Environ. 1997; 32(1):7-11. doi: https://doi.org/10.1076/ snfe.32.1.7.13462

17. Arroyo-Arce S, Thomson I, Fernández C, Salom-Pérez R. Relative abundance and activity patterns of terrestrial mammals in Pacuare Nature Reserve, Costa Rica. Cuadernos de Investigación UNED. 2017; 9(1):15-21. https://doi.org/10.22458/urj. $\underline{\text { vili.1673 }}$

18. Ramirez-Mejia A, Sánchez F. Activity patterns and habitat use of mammals in an andean forest and a eucalyptus reforestation in Colombia. Hystrix. 2016: 27(2):1-7. https:// doi.org/10.4404/hystrix-27.2-11319

19. Caceres N, Monteiro-Filho E. Food habits, home range and activity of Didelphis aurita (Mammalia, Marsupialia) in a forest fragment of southern brazil. Stud Neotrop Fauna Environ. 2001; 36(2):85-92. https://doi. org/10.1076/snfe.36.2.85.2138

20. Maffei L, Noss A. How small is too small? Camera trap survey areas and density estimates for Ocelots in the Bolivian Chaco. Biotropica. 2008.; 40(1):71-75. https://doi. org/10.1111/j.1744-7429.2007.00341.x 